\title{
Nuevos retos en la investigación del contenido léxico: elementos para una neurosemántica*
}

\author{
New challenges in lexical content research: \\ keys for a neurosemantic approach
}

\section{CARMEN VARO VARO}

RECIBIDO: 1 DE JULIO DE 2016

Grupo de investigación "Semaínein" ACEPTADO: 16 DE FEBRERO DE 2017

Instituto Universitario de Investigación en Lingüística Aplicada

Área de Lingüística General

Departamento de Filología

Facultad de Filosofía y Letras

Universidad de Cádiz

Avda. Gómez Ulla, nº 1

11003 Cádiz-España

carmen.varo@uca.es

Resumen: La investigación surgida en las últimas décadas en los ámbitos de la psicolingüística y la neurolingüística sobre el procesamiento semántico hacen necesaria una revisión de algunas de las aportaciones del paradigma metodológico estructuralista, destinada a alcanzar una formulación más flexible de la configuración del significado léxico. El presente trabajo parte de una concepción de este como red funcional con base psiconeurológica derivada del estrecho vínculo entre los aspectos cognoscitivos y semánticos del léxico. Nos proponemos 1) abordar con más detalle dicha conexión, sin renunciar a su delimitación, y 2) examinar algunas evidencias concretas derivadas del procesamiento en este nivel, junto con los principales datos sobre su organización cerebral. Todo ello nos conduce a una nueva dimensión neurocognitiva del contenido léxico en el marco de las actuales orientaciones de la Semántica léxica, en la que deben incorporarse, sin duda, los diferentes procesos y mecanismos implicados en la producción y comprensión del significado.

Palabras clave: Significado léxico. Neurosemántica. Procesamiento semántico. Contenido léxico. Estructura semántica.

\footnotetext{
* Este trabajo se enmarca en el Proyecto I+D "Comunicación especializada y terminografía: usos terminológicos relacionados con los contenidos y perspectivas actuales de la semántica léxica" (ref. FFI2014-54609-P), financiado por el Programa estatal español de Fomento de la Investigación Científica y Técnica de Excelencia (subprograma estatal de generación del conocimiento), en el marco del Instituto Universitario de Investigación en Lingüística Aplicada, Universidad de Cádiz (ILA).
} 
Abstract: Research on semantic processing in psycholinguistics and neurolinguistics over the last decades makes necessary a partial revision of the structural linguistic methodological paradigm. The revision aims at achieving a more flexible formulation of the lexical meaning structure. On the grounds of a conception of meaning as a functional network with a neuropsychological base emerging from the close link between semantic and cognitive parts of the lexicon, this paper intends 1) to address in more detail these related parts, and 2) to discuss evidence derived from an analysis of the processing at this level, along with the major facts we know about the internal organization of our brain. All this leads to the description of a new neurocognitive dimension of lexical content within the framework of lexical semantics.

Keywords:Lexical meaning. Neurosemantics. Semantic processing. Lexical content. Semantic structure.

\section{INTRODUCCIÓN}



os numerosos problemas surgidos dentro del estructuralismo lingüístico en la explicación de la naturaleza del significado léxico y del funcionamiento de las relaciones semánticas, sobre todo derivados de la exclusión en dicho marco teórico de los procesos de codificación y descodificación en los que los contenidos significativos participan, propiciaron, sin duda, el auge de nuevos enfoques teóricos como la lingüística cognitiva en la investigación del léxico de las lenguas, así como el consiguiente abandono progresivo de una metodología de trabajo que había sido enormemente productiva durante décadas.

En efecto, el análisis en términos de rasgos semánticos o análisis componencial, aplicado inicialmente en fonología y con posterioridad trasladado a la indagación de los campos léxicos, fue adoptado por muchos lingüistas, si bien estos semas o entidades de contenido mínimo resultaban más complejos que los rasgos distintivos fonológicos, ya que conjugaban aspectos funcionales o de lengua y rasgos enciclopédicos o derivados de la experiencia real de los hablantes. Este tipo de análisis proporcionaba una descripción del significado generalmente bien delimitada frente al concepto, en la que los rasgos diferenciales, sobre una base semántica común, constituían la clave que permitía la caracterización de cada unidad en el conjunto del sistema. Sin embargo, también fue usual objeto de críticas, dirigidas en especial a la problemática noción de privatividad establecida en él (ver Varo Varo 2010, 151-52) y a la rigidez de sus planteamientos, que apenas dejaba lugar para aclarar el funcionamiento de determinados fenómenos del hablar en los que desempeñan un importante papel los "sentidos", para cuyo análisis se hace necesario el conocimiento de ciertos factores de orden pragmático que rodean al proceso comunicativo. 
Frente a él, el modelo de análisis proporcionado por el paradigma cognitivo, partidario de la dependencia de las categorías lingüísticas respecto a la organización conceptual y los mecanismos de procesamiento, integraba el significado en el complejo proceso de la comunicación y lo vinculaba con procesos mentales como la percepción, la categorización, la memoria y la atención. Según este, el lenguaje forma parte de un complejo sistema cognitivo en el que actúan diversos mecanismos de conceptualización basados fundamentalmente en la separación entre el individuo y el entorno que le rodea, el conocimiento relativo a la manipulación de objetos, la experiencia espacial y el control de los movimientos.

No obstante, la falta de delimitación entre aspectos conceptuales y aspectos netamente lingüísticos, la visión gradual del significado (frente a la tradicional interpretación discreta de los rasgos semánticos), junto a la falta de consenso en los modelos vigentes de producción y comprensión léxicas, impiden, a nuestro juicio, avanzar en la caracterización y singularización de la noción de significado léxico y los procesos de construcción de este. Así, mientras que se asume con naturalidad que el significado oracional es un constructo derivado de la interrelación de múltiples tipos de información, se tiende a identificar el significado léxico con un contenido acabado que puede ser recuperado a través de distintas vías de acceso.

La investigación surgida en las últimas décadas en los ámbitos de la psicolingüística y la neurolingüística sobre el procesamiento semántico hacen necesaria una revisión de algunas de las aportaciones del paradigma metodológico estructuralista, que nos permita alcanzar una formulación más flexible, aunque no por ello menos rigurosa, de la naturaleza y la estructura del significado léxico, en la que es posible retomar el enfoque componencial como un proceso de reconstrucción de la configuración semántica de la palabra. En este sentido, asumiendo una concepción de significado como red funcional de base neurológica, derivada del estrecho vínculo entre los aspectos cognoscitivos y semánticos del léxico y tomando en consideración las nuevas orientaciones de la Semántica léxica, en las que deben incorporarse los diversos procesos y mecanismos de la comprensión y la producción del significado, los objetivos fundamentales de este trabajo son: 1) abordar con más detalle las múltiples conexiones del contenido léxico con los principales procesos neurocognitivos participantes en el procesamiento de la información lingüística por parte del individuo, y 2) examinar algunas evidencias proporcionadas por la indagación del procesamiento de este nivel, junto con algunos datos sobre su 
organización cerebral, que nos conducen a un cambio de perspectiva no solo en los modelos neuropsicológicos del lenguaje, sino también en los estudios lingüísticos, en general, y léxicos, en particular.

\section{LA NATURALEZA DEL SIGNIFICADO LÉXICO. LA PRODUCCIÓN EN INDIVI- DUOS CON DÉFICITS SEMÁNTICOS}

Uno de los rasgos más sobresalientes de la Semántica léxica de corte estructuralista es su reivindicación del significado como objeto de estudio diferenciado del concepto, frente a otras corrientes como la generativista y la cognitivista, que renuncian a ello. En esta línea, décadas de investigación han tratado de establecer con la mayor exactitud posible los diversos niveles ${ }^{1}$ del "significar", con la intención de erigir al significado en objeto científico que puede ser abordado desde una perspectiva funcional (ver Casas Gómez 2014).

En lo que concierne a los modelos del procesamiento léxico, pese a la atención dirigida por parte de los estudiosos hacia la detallada segmentación de las diversas fases de la producción y la comprensión léxicas, en general, en muchas de las aproximaciones tanto neurolingüísticas como cognitivas no parece que se establezcan límites precisos entre las categorías cognoscitivas y las semánticas. Así, podemos mantener que, mientras que las primeras representan propiedades del entorno que rodea al individuo, las segundas se apoyan en las otras para marcar diferencias lingüísticas.

Si retornamos al desiderátum estructuralista, sería esperable encontrar evidencias empíricas en las investigaciones actuales sobre las bases psicobiológicas del lenguaje que permitan dilucidar si es posible deslindar de las relaciones conceptuales, sin renunciar a su origen cognitivo, las relaciones establecidas, en el seno de los sistemas léxicos particulares, entre significados de signos

1. Si nos atenemos a la delimitación de niveles del significar o tipos de "contenido" trazada desde la semántica estructural, mientras que el "significado", definido como contenido formalizado intralingüísticamente en las lenguas particulares, supone la base semántica del análisis estructural y funcional de las lenguas, el "sentido" constituye un contenido lingüístico especial que aflora en el texto, como resultado de la interacción de los signos con nuestros conocimientos del mundo. Tal caracterización de "significado" y "sentido" permite asignar el primero a la lingüística de la lengua, y ubicar el segundo en el ámbito más amplio de la lingüística del hablar. Una revisión y aplicación de estos niveles del significar puede verse en Gutiérrez Ordóñez (1981 y 1989) y Casas Gómez (1995). Uno de los modelos actuales más completos es el propuesto por Casas Gómez (1999 y 2002), que, de acuerdo con los dos niveles del significar y los dos tipos de lingüística, distingue "sentido" y "denotación" ("designación real” o "referencia"), frente a "significado" y "designación" ("potencial” o "designación de lengua"). 
que comparten un fondo sémico común y presentan una serie de rasgos diferenciales, y cuya diversa articulación depende de la organización concreta de dichos sistemas. ${ }^{2}$ Dentro de este marco, reseñaremos en este apartado algunos datos y observaciones procedentes del estudio del comportamiento verbal de individuos afectados por déficits semánticos, en particular la afasia anómica y las demencias semánticas, desde el punto de vista de la producción léxica, por su interés para la interpretación del componente semántico del lenguaje.

La anomia pura, ${ }^{3}$ considerada como una modalidad de afasia en la que es particularmente notable la incapacidad para la recuperación nominal en el caso de conceptos cuya representación léxica ha sido plenamente adquirida y usada con anterioridad, proporciona una fuente de información de incuestionable valor para la investigación sobre la organización interna de los sistemas perceptivo y lingüístico y su interrelación durante el procesamiento léxico. Los pacientes con esta disfunción, según se desprende de los datos proporcionados por los estudios sobre esta afasia (ver Varo Varo 2014 y 2015), se caracterizan, en primer lugar, por la ausencia de dificultades desde el punto de vista de la recuperación conceptual de los referentes (incluida su integración categorial). Por otra parte, al no presentar problemas de fluidez, los anómicos pueden acceder a distintas opciones expresivas, ${ }^{4}$ como los circunloquios y las palabras ómnibus, como "tijeras" = 'algo para coser', "bolso" = 'lo que uso para comprar' o "pluma” = 'especie de lápiz' (ver Ellis 130-31), si bien también son comunes las parafasias semánticas o sustituciones por elementos léxicos de la misma esfera significativa, ${ }^{5}$ vinculados por muy dispares tipos de relación, como "enganche" por "hebilla", "persona" por "niño", "hierro" por "llave", "calendario" y "reloj" por "cronómetro", "cierre" por" ojal", "cadena" por "correa" y "teléfono" por "televisor (ver Diéguez Vide y Serra-Casanova 288 y 290). En segundo lugar, aunque, en líneas generales, son capaces de rechazar nombres incorrectos, esta

2. En relación con este propósito, la tipología de relaciones semánticas recientemente propuesta por Casas Gómez (2014) constituye una importante base teórica para abordar tanto terminológica como conceptualmente las diferentes distinciones posibles en el ámbito del análisis semántico.

3. Goodglass y Wingfield definen la anomia como "a difficulty in finding high information words, both in fluent discourse, and when called upon to identify an object or action by name" (3). Para una caracterización y propuesta de tipologización de la anomia, puede verse Cuetos y Diéguez Vide y Peña-Casanova (296-306).

4. Diéguez Vide y Peña-Casanova (286-92) recogen una completa clasificación de los tipos de respuestas en anómicos.

5. Tales observaciones contrastan con la ausencia de parafasias fonémicas, presentes en las afasias de Broca, Wernicke y de conducción (ver Varo Varo 2015). 
habilidad se ve reducida cuando se trata de unidades léxicas semánticamente cercanas, ${ }^{6}$ ya se trate de parasinónimos, cohipónimos o hiperónimo-hipónimo, lo que apunta hacia un error de concreción sémica, previa a la completa recuperación fonológica, teniendo en cuenta que el reconocimiento conceptual de tales objetos no está afectado. En tercer lugar, la coincidencia en la preservación del léxico funcional (es decir, elementos de valor meramente gramatical) y del más frecuente nos revela que la dificultad no estriba en el tipo de léxico sino en la operación mental requerida por cada unidad, ligada a la experiencia, variable de un individuo a otro, en cuanto a las conexiones entre las marcas semánticas que conforman su significado.

También el síndrome conocido como demencia semántica ${ }^{7}$ se caracteriza, como la anomia, por la pérdida de la "memoria de las palabras" en la producción (ver Varo Varo 2015), si bien se añaden en ella importantes dificultades en la comprensión, inicialmente circunscritas al léxico menos frecuente (ver Hodges y Patterson) y generalmente subsanadas por el marco sintáctico y pragmático, por lo que suelen pasar desapercibidas para los familiares de los afectados. En efecto, el deterioro en la comprensión y denominación léxicas que este lleva aparejado (ver Snowden, Goulding y Neary) atañe a la memoria semántica y se manifiesta en forma de afasia fluente, con un discurso vacío, pérdida gradual del significado léxico, parafasias semánticas y progresiva sustitución de nombres específicos por nombres genéricos (ver Farah y Grossman; Hodges y Paterson; Donoso y otros) como se pone de relieve en expresiones del tipo "es un lugar de un hogar", "es algo para quebrar...no es un cuchillo... algo que corta" o "no traje mi reloj" para referirse a "habitación", "serrucho" y “reloj”, respectivamente (Donoso y otros 283).

Así pues, ambas disfunciones muestran semejantes resultados en tareas de decisión entre el nombre correcto y otros, ${ }^{8}$ la preservación del léxico fun-

6. Allport (76) comenta, entre sus casos clínicos, el de una paciente que manifestaba dudas al decidir entre las palabras "clavo" y "tornillo", muy cercanas desde el punto de vista de su significado, para una imagen del primero, en tanto que se mostraba segura al descartar respuestas que corresponderían a otras esferas semánticas.

7. La denominación "demencia semántica" es acuñada por Snowden, Goulding y Neary para describir tres pacientes afectados de afasia progresiva primaria, con déficits de comprensión léxica, además de en el conocimiento de objetos y personas. Para algunos la demencia semántica constituye, en realidad, una variante temporal de la demencia fronto-temporal (ver Hodges y Patterson).

8. Aunque el factor "familiaridad" es compartido (Lambon Ralph y otros 782; Bozeat y otros 1211), parece que los pacientes con demencia semántica obtienen mejores resultados en las pruebas asociativas de imágenes que en las de asociación entre palabra y significado (ver Bozeat y otros), lo que puede obedecer al diferente tipo de vínculo requerido en cada prueba. Mien- 
cional, la necesidad de cierta especificación semántica previa para que se inicie la recuperación fonológica y, sobre todo, las frecuentes parafasias semánticas, presentes tanto en el discurso espontáneo como en las respuestas para la denominación de referentes, en contraste con la ausencia de parafasias fonéticas. Además, entre todos los factores valorados en la bibliografía como elementos implicados en el proceso de denominación a partir de tales conductas verbales, en ambos casos tienen singular relevancia la familiaridad con el referente, la frecuencia y la edad de adquisición, lo que puede refrendar un modelo de organización de la información semántica en el que el número de rasgos y la robustez de las conexiones entre ellos, reforzada por los anteriores factores, servirá para predecir el éxito en la producción léxica de tales pacientes (Lambon Ralph y otros 778). Para dar contestación a estos datos, se han perfilado propuestas a favor de la ordenación jerárquica del conocimiento léxico, de acuerdo con los tradicionales modelos de memoria semántica, sobre la base de ciertas sustituciones por hiperónimos (por ejemplo, "animal" por "caballo"). Sin embargo, no se trata de la respuesta más común en anómicos o en afectados de demencia semántica, pues son habituales todo tipo de asociaciones semánticas, como la cohiponimia (por ejemplo, "teléfono" por "televisor"), metonimia (por ejemplo, "hierro" por "llave"), etc. Además, encontramos respuestas similares a las que se producen en algunos errores espontáneos del habla no patológica y en los estados TOT. Asimismo, se ha de considerar la probable mayor frecuencia de los sustitutos.

Centrándonos en las diferencias entre ambas disfunciones, frente a la anomia, el deterioro de la demencia semántica se extiende más allá del significado léxico y afecta, aunque inicialmente en menor medida, al conocimiento conceptual. Podríamos, por tanto, decir que, en tanto que la dificultad de los pacientes anómicos radica en el lazo semántico proyectado, en el caso de los pacientes que padecen demencia semántica el problema reside en la conformación del lazo conceptual, en el que queda incluido el primero. De esta forma queda patente la relación entre significado lingüístico e información conceptual, expresada en distintos grados de implicación en cada una de estas afecciones (ver Varo Varo 2015). La propia evolución en las respuestas en tareas de denominación producidas por enfermos con demencia semán-

tras que la relación entre palabra y significado es arbitraria, las imágenes se benefician más del conocimiento conceptual sobre el objeto. 
tica, desde términos específicos hasta elementos ómnibus ("cosa", "hacer", etc.) e incluso omisión de respuesta, ilustra perfectamente la extensión de su incidencia desde la esfera de la integración de rasgos netamente lingüísticos, pasando por el campo más abierto de las relaciones conceptuales, hasta llegar a desaparecer ambos, con el consiguiente fracaso de la recuperación léxica. ${ }^{9}$

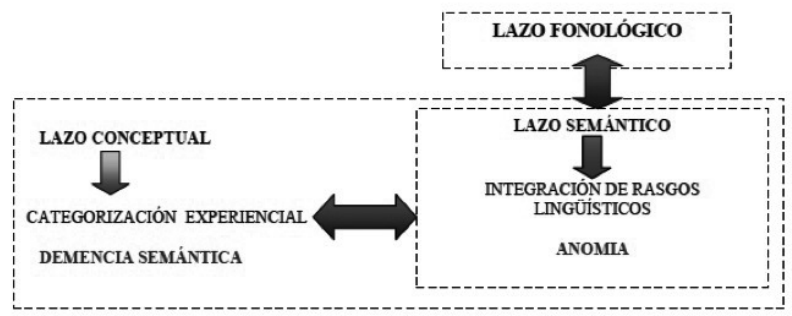

Interacción entre los ámbitos conceptual, semántico y fonológico

Por lo que respecta a la naturaleza del área semántico-conceptual del significado léxico, los modelos elaborados a partir de los datos procedentes de enfermedades neurodegenerativas como la demencia semántica ponen de relieve que la función lingüística no es responsabilidad de regiones aisladas sino de la conectividad tanto anatómica como funcional de un sistema altamente interactivo y advierten la existencia de diferentes "corrientes" anatómicas ubicadas en las regiones dorsal y ventral del hemisferio izquierdo e implicadas en el lenguaje, en consonancia con el modelo propuesto por Hickok y Poeppel (2004 y 2007). De acuerdo con esta teoría, mediante la valoración en vivo de las vías de sustancia blanca vinculadas con el lenguaje en pacientes con demencia semántica a través de la combinación de la tractografía por tensor de difusión (TTD) y la resonancia magnética (IRMf) (ver Gorno-Tempini y otros; Agosta y otros) se ha detectado una alteración en el camino ventral en el que participan estructuras como el fascículo lateral inferior y el lóbulo temporal anterior, en contraposición con la relativa conservación de la vía dorsal fronto-parietal, responsable de

9. Algunos ejemplos de deterioro de la red semántica-conceptual recogidos por Hodges y Patterson (1006) ilustran la pérdida de las asociaciones semánticas, inicialmente basadas en la sustitución por cohipónimos o hiperónimos más frecuentes, hasta limitarse a meras conexiones conceptuales e incluso incapacidad de recuperación conceptual: "elephant"> "horse"> "animal"; "chicken"> "bird"> "animal"; "orange"> "apple"> "for food"; "scissors"> to cut things">"a machine"; "violin"> "music"> "don't know". 
la gramática y los procesos fonológicos subléxicos. Tales hallazgos nos brindan una formulación más flexible de la naturaleza y estructura del significado, como red funcional de base neurológica, frente a la interpretación modular tradicional, y en la que es posible retomar la perspectiva composicional como un proceso de reconstrucción de la configuración semántica de la palabra.

\section{LA CONSTRUCCIÓN DEL CONTENIDO LÉXICO}

\subsection{Propuestas teóricas de base neurocognitiva}

Aunque los datos más recientemente proporcionados, gracias al empleo de las nuevas técnicas de neuroimagen, apuntan con claridad hacia la existencia de redes neuronales interconectadas del conocimiento en las lenguas, la falta de unanimidad en los modelos psicolingüísticos sobre la organización de la información semántica sigue siendo notable. En este marco, entendemos que el individuo durante el procesamiento no opera con unidades prealmacenadas, ${ }^{10} \mathrm{a}$ las que se tiene acceso en función del tipo de palabra, sino que se apoya en un mecanismo de reconstrucción léxica, basado en el análisis lingüístico secuencial y orientado por aspectos como la frecuencia y el contexto. Un importante correlato neurológico en relación con los actuales avances en neurociencia cognitiva es el enfoque de la web funcional (ver Lamb 1999 y 2004; Pulvermüller 2001, 2005, 2012 y 2013; Kiefer y Pulvermüller, entre otros), entendida como un conjunto de neuronas, distribuidas sobre diversas áreas corticales, dependientes entre sí y que trabajan como una unidad funcional. Tales redes se extienden tanto por la corteza perisilviana como por otras áreas corticales distantes, por lo que se excluye la interpretación tradicional de significado como centro unificado de contenido. Esta propuesta cuenta con importantes precedentes en la bibliografía anterior como la noción de asamblea celular, introducida por $\mathrm{Hebb}^{11} \mathrm{y}$ ha sido elaborada bajo múltiples variantes, entre las que destaca la no-

10. La hipótesis del almacenamiento de información simbólica, compartida por gran número de investigadores de distintas tendencias, ha sido especialmente criticada desde el marco de lingüística neurocognitiva por Lamb $(1999,375)$, que en relación con esta cuestión señala: "The mind is not a device for storing and rewriting symbols but a network system, whose information is in its conectivity".

11. La denominación "cell asembly", acuñada por Hebb $(60-78,196)$ y referida a toda una red neuronal extendida que puede ser activada en su conjunto de manera reiterada durante ciertos procesos mentales, representó, en cuanto a su planteamiento, una postura de consenso entre las posiciones holista, defensora de la equipotencialidad de las distintas regiones cerebrales en cualquier actividad cognitiva, y las posiciones excesivamente localizacionistas, tendentes al 
ción de atractor del procesamiento conceptual (ver Cree, McRae y McNorgan), entendida como red entrenada para procesar partiendo de la forma léxica las representaciones semánticas basadas en rasgos generados por el sujeto. En ese marco, tanto la unidad léxica como los contenidos semánticos a ella asociados no se limitan a una zona limitada y específica del cerebro, sino que se extiende por amplias áreas corticales relacionadas con el procesamiento de información perceptiva, motora e incluso emotiva, con distinto grado de relevancia en función de la tarea cognitiva en cuestión.

La actuación de la dimensión semántica como interfaz entre lenguaje y cognición, que permite abordar el contenido léxico a la luz de distintos mecanismos mentales con los que interactúa, entre los que se hallan la percepción, la memoria o la atención, nos conducen al debate sobre los límites de la red semántica. Así, de tales interacciones surgen diversos modelos explicativos del significado, como los inspirados en la organización de la memoria (ver Ullmann; Kutas y Federmeier), que vinculan el contenido léxico fundamentalmente a la memoria declarativa, si bien también reconocen su implicación en la memoria de trabajo; los inspirados en el funcionamiento de la atención y la consciencia, entre los que podemos singularizar la semántica atencional (ver Marchetti; Marchetti, Benedetti y Alharbi), que explica los procesos significativos desde la teoría de la mente, pero, sobre todo, queremos destacar, por su gran calado en la investigación actual, las propuestas inspiradas en los procesos de percepción-acción en los que se ve envuelto el individuo, como contrapunto al tradicional modelo modular del significado.

La denominada semántica corporeizada cuenta con diversas formulaciones, desde las más radicales, que asumen que el lenguaje está entretejido en la acción y la percepción desde el punto de los circuitos cerebrales (Barsalou 1999 y 2008; Glenberg y Gallese), hasta las más comedidas, como la de Caramazza y Mahon (354-67), que acepta la existencia de un nivel de contenido conceptual no constituido por información sensorial y motora. Pulvermüller (2012 y 2013) ofrece una propuesta integradora que llega a distinguir, atendiendo a los procesos mentales y estructuras cerebrales implicadas, hasta cuatro tipos de facetas de la Semántica: 1) semántica referencial, que esta-

encapsulamiento de las funciones neurológicas. El respaldo de los actuales datos proporcionados por la neuroimagen y otras técnicas para el análisis del procesamiento de la información (registro eléctrico, o Potenciales Evocados Relacionados con Eventos (ERP) y magnético, o Magnetoencefalografía (MEG), entre otras) convierten a esta propuesta en una de las principales precursoras de la neurociencia contemporánea. 
blece vínculos entre símbolos y objetos y acciones (áreas motoras, sensoriales y de integración interconectadas); 2) semántica combinatoria, que permite el aprendizaje del significado simbólico a través de la coocurrencia en contextos (corteza perisilviana); 3) semántica afectiva-emotiva, que vincula signos con estados internos del individuo (áreas de interconexión entre corteza perisilviana y sistema límbico), y 4) semántica abstracta, relativa al conocimiento de entidades abstractas y mecanismos de generalización (corteza prefrontal, temporal anterior y parieto-temporo-occipital). Si aplicamos este modelo de análisis a una unidad léxica como "resbalar", por ejemplo, podríamos deslindar, siguiendo el orden aquí propuesto: 1) los aspectos relativos a la experiencia sensoriomotora en relación con este verbo, situada en una dimensión referencial; 2) su combinatoria de lengua, creada a partir de la experiencia lingüística del individuo; 3 ) los vínculos emotivos establecidos desde la perspectiva experiencial del individuo en relación con esa unidad léxica, y 4) por último, el contenido abstracto compartido con el resto de hablantes de la lengua en virtud de los procesos cognitivos de generalización a partir del uso continuado de esa unidad.

Ahora bien, aunque se reconoce la existencia de redes relacionadas con el significado, de acuerdo con las aproximaciones neurolingüísticas, con base en el lóbulo temporal inferior y la confluencia temporo-parieto-occipital izquierda (ver Binder y otros), no hay un único modelo de la representación semántica. Para los más tradicionales el conocimiento conceptual es representado en un formato amodal, como zona de convergencia, que se nutre de múltiples conocimientos de modalidad específica, especialmente percepción y movimiento, si bien está separado de ellos (almacén conceptual unitario) (ver, como muestra, Rumelhart y McClelland; Caramazza y otros 1990). Este sustrato compartido, derivado de la actividad interactiva de las representaciones emanadas de todas las modalidades, tiene como ventaja permitir el acceso a gran cantidad de información a partir de tan solo un fragmento de una modalidad ${ }^{12}$ (ver Patterson, Nestor y Rogers; Hodges y Patterson). En

12. Ahora bien, considerando el enorme coste cognitivo que conllevaría el acceso y la recuperación de la memoria léxica de toda la información asociada a cualquier entrada, acompañado del consiguiente aumento del tiempo de procesamiento, debemos resaltar el especial protagonismo durante el procesamiento lingüístico de la habilidad cognitiva de la atención, que permite atender a partes de nuestra experiencia relevantes para un determinado propósito al tiempo que son ignorados otros aspectos irrelevantes para tal propósito (Croft y Cruse 6). De aquí puede extraerse la necesidad de ahondar en la función de este mecanismo en el procesamiento léxico. 
contraposición, encontramos cada vez más modelos inspirados en representaciones conceptuales distribuidas y corporeizadas, que atribuyen mayor protagonismo a los sistemas sensorial, motor y emocional para dar cuenta de los procesos semánticos (ver Kiefer y Pulvermüller). El problema que permanece sin aclarar es el de la delimitación del significado lingüístico en relación con la esfera conceptual.

Por otra parte, el hallazgo de sustratos cerebrales compartidos por funciones lingüísticas y otras funciones cognitivas como la memoria ha potenciado modelos de la organización del conocimiento (ver Ullmann; Kutas y Federmeier), que vinculan el contenido léxico fundamentalmente a la memoria declarativa, en tanto que los aspectos relativos a su configuración formal se adscriben a la memoria procedimental, lo que plantea la duda sobre la existencia de subordinación o solapamiento de funciones. En cualquier caso, estas conexiones no solo sirven de base explicativa de la separación entre los aspectos semánticos y formales del léxico, ya mencionada, sino que también nos justifica las indudables implicaciones de la memoria de nuestras múltiples experiencias (sensoriales, motoras, emocionales) desde el punto de vista del enriquecimiento contextual durante el procesamiento léxico. Sin duda, planteamientos de este tipo merecen la reflexión y, tal vez, la profundización, por parte de las teorías semánticas del contenido léxico.

\subsection{Algunas evidencias a partir de la neología semántica}

En lo que concierne a los datos disponibles, la organización semántico-conceptual de la información léxica puede ser abordada desde el prisma de la neología semántica, puesto que afecta a unidades fonológicamente conocidas, es decir, supuestamente registradas en el lexicón mental, pero cuyos significados no se corresponden con el conjunto de contenidos habitualmente asociados a estas entradas. ${ }^{13}$ De ahí que, entre los posibles modelos explicativos de este fenómeno, se adecue mejor a los de activación extendida y búsqueda en paralelo $^{14}$ (Morton 1969 y 1979), en los que se incluyen estrategias cognitivas

13. Como hemos puesto de relieve en trabajos anteriores (ver Díaz Hormigo y Varo Varo), también son notables las limitaciones en los modelos explicativos de la comprensión y la producción léxicas a la hora de dar cuenta de las creaciones neológicas.

14. En cambio, la neología formal encaja mejor en los modelos seriales de búsqueda y acceso múltiple (ver Forster y Chambers), ya que en ellos las representaciones morfológicas forman parte de la memoria léxica y, como consecuencia, pueden orientar el proceso de construcción del significado de la nueva unidad. 
que facilitan la asociación semántico-conceptual y la interacción del contexto, precisadas por las metáforas.

En el reconocimiento de significados de nueva creación, ${ }^{15}$ se ha sugerido que las propias rutas metafóricas ya conocidas facilitan, sin un gran coste interpretativo, la activación de nuevas conexiones (ver Shibata y otros; Thibodeau y Durgin). En esta línea, es bien conocida la explicación de los procesos metafóricos ofrecida desde hace años por la lingüística cognitiva como proyección selectiva de las propiedades ${ }^{16}$ de un dominio de partida sobre un dominio meta (ver Lakoff y Johnson; Lakoff; Rivano). Asimismo, se ha señalado que, durante el procesamiento, mientras que las propiedades asociadas son activadas, otras irrelevantes son inhibidas para que no interfieran en el acceso (ver McGlone y Manfredi).

Haciendo balance crítico, queda, sin embargo, por verificar si se ha de acceder primero a los significados canónicos completos ya conocidos para poder identificar los nuevos, lo que supone, al menos de manera implícita, una concepción más o menos cerrada de las significaciones, o es suficiente con activar algunos de los rasgos, no necesariamente nucleares del contenido léxico. Por otra parte, la mayoría de las aproximaciones tanto cognitivas como neurolingüísticas, desde sus diferentes bases teóricas, coinciden en reconocer el acceso a múltiples representaciones activas de manera conexa en cada fase del procesamiento. No obstante, la asunción de que "accedemos" a una representación semántica o la "recuperamos"17 induce a la contemplación del significado como una entidad que es seleccionada de entre un conjunto de posibilidades dadas de antemano. Por último, para afrontar las limitaciones de los modelos clásicos de la comprensión y la producción léxicas a la hora de dar

15. Se ha de tener presente la separación entre metáfora conocida y neología semántica. Así, aunque se ha demostrado mediante estudios experimentales que no hay diferencia en términos de duración temporal o esfuerzo cognitivo en el procesamiento de significados convencionales frente a significados metafóricos (ver Pollio y otros), los resultados son distintos para las metáforas de nueva creación (ver Geiger; Arzouan y otros; Lai, Curran y Menn). Sobre las bases biológicas de las metáforas de nueva creación, ver Bottini y otros; Pynte y otros; Geiger; Schmidt y otros; Ahrens y otros; Mashal y otros, Arzouan y otros; Lai, Curran y Menn.

16. Para explicar la conexión entre significado de lengua y significado metafórico, han surgido modelos como el de inclusión de clases de Glucksberg (92-96), en el que se contempla cierta vinculación polisémica entre ambas. Esta propuesta se ajusta mejor a la metáfora convencional que a las creaciones neológicas de naturaleza semántica, pues el vínculo entre los dos significados implicados es muchas veces bastante débil.

17. A propósito de este enfoque tan frecuentemente aceptado en la investigación psicolingüística, en la línea de otros autores (ver Bennett y Hacker 44), debemos cuestionarnos nociones como las de "representación" y "acceso" como elementos clave en los modelos explicativos del procesamiento léxico. 
cuenta de las creaciones neológicas y, en suma, trazar un modelo explicativo plausible del procesamiento léxico (ver Varo Varo, Díaz Hormigo y Paredes Duarte; Díaz Hormigo y Varo Varo; Varo Varo 2013) son necesarias tanto la profundización en los principales factores que participan en la interpretación de enunciados que incorporan neologismos y los mecanismos cognitivos esenciales implicados en el procesamiento de estas unidades, con objeto de calibrar el grado de eficiencia y la interacción de estos, como la búsqueda de correlatos biológicos que avalen los efectos experimentales hallados por la investigación psicolingüística.

En cuanto a la primera de estas cuestiones, la aproximación a la neología a través de tareas de decisión léxica nos permite recabar información sobre la forma de procesamiento y el esfuerzo cognitivo invertido, lo que contribuye a la caracterización de significado desde una perspectiva psicolingüística. Entre los efectos experimentales que hemos presentado en investigaciones anteriores (ver Varo Varo 2013), se halla en primer término la frecuencia, factor que ha sido ampliamente tratado en la bibliografía sobre procesamiento léxico (ver Bradley y Forster), puesto que, además de afectar al conjunto de la palabra, concierne a los constituyentes de manera desigual (ver Varo Varo 2013). De manera secundaria, son destacables los efectos de facilitación, la superioridad de la palabra (ver Burani y Caramazza), la similitud con otras, pero, sobre todo, la disponibilidad contextual o facilidad que un sujeto tiene para imaginar un contexto o circunstancia concretos en los que pudiera ocurrir la palabra que se pretende reconocer.

Respecto a los mecanismos cognitivos observados en las pruebas de reconocimiento de neologismos, la mayoría de los participantes logran interpretar adecuadamente los enunciados en que aparecían insertos. Puede, por tanto, decirse que el enorme poder apelativo de las tales creaciones conduce al individuo a la búsqueda de rutas de reconstrucción léxica que pueden diferir de las establecidas para el procesamiento de unidades ya conocidas. En relación con ello, debemos señalar la implicación en la interpretación léxica de un proceso de activación de vínculos semántico-conceptuales a través de la selección de rasgos, que convive e interacciona con el proceso de integración de la estructura formal de la palabra, como se desprende de las pruebas experimentales de facilitación. La interrelación de ambos mecanismos se evidencia también en que no se incrementa la dificultad de comprensión en las formaciones en las que, además de un proceso de derivación o composición formal, se da un proceso metafórico, como en el caso de "dinerofagia" ('codicia') (ver Varo Varo 2013), que incluso 
se interpreta con mayor facilidad que otros neologismos formales en los que no hay proceso metafórico. Por otra parte, el acierto en el procesamiento de las creaciones semánticas nos hacen pensar que estas no son interpretadas a través del significado convencional completo, sino que son solo ciertos rasgos semánticos los que facilitan la reconstrucción del significado, con lo que se reduce considerablemente el esfuerzo cognitivo y, como consecuencia, el tiempo invertido. Si consideramos los enunciados siguientes, en los que aparecen unidades neológicas,

1. Pero ello no *agrieta mi argumento fundamental. (CREA. Ussía, Alfonso. Tratado de buenas maneras. Vol. 2. Barcelona: Planeta, 1994).

2. Bárbara andaba detrás de la historia, con esa hambre de noticia que sólo tienen los *purasangre del periodismo. (CREA. Rojo, Alfonso. $M a-$ tar para vivir, Barcelona: Plaza Janés, 2002).

durante su procesamiento no es imprescindible acceder al contenido canónico de "agrietar" y "purasangre", sino simplemente a algunas de las marcas asociadas a ambos signos favorecido por un marco cognitivo que inhibe otras marcas semánticas a las que en otros contextos también se suele propagar la activación ('aplicado a cuerpos sólidos' y 'aplicado a caballos').

Para terminar, desde el punto de vista de sus bases biológicas, el procesamiento de neologismos semánticos ha sido singularizado, frente al de unidades léxicas de significado literal e incluso de metáforas convencionales, por la actuación de mecanismos propios de un análisis holista, que requiere del reclutamiento de regiones cerebrales ubicadas fuera de los circuitos lingüísticos centrales. Se ha señalado, en esta línea, la participación del hemisferio derecho en la producción y la comprensión de nuevos significados, ${ }^{18}$ en consonancia con los efectos experimentales expuestos en el apartado cuarto. No obstante, en tales casos, debe delimitarse con claridad la esfera de influencia del nuevo significado y profundizarse en la interfaz léxico-sintaxis.

Llegados a este punto, podemos confirmar a través del acercamiento tanto a los déficits semánticos como al fenómeno de la neología, que la conformación del conocimiento conceptual no es estática, sino que se ve cons-

18. El procesamiento de neologismos semánticos, característico del hemisferio derecho, imprescindible para la producción y la comprensión de nuevos significados léxicos, ha sido analizado, a partir del estudio de pacientes afásicos, por Marangolo y otros (364-71). 
tantemente alterada por la participación de la memoria episódica para acabar determinando la memoria semántica, más estable, en virtud de la experiencia repetida. Desde la perspectiva de la comprensión léxica, parece evidente que el significado no puede concebirse como una entidad encapsulada sino como una red dinámica derivada de un proceso de construcción semántica en el que influyen tanto aspectos lingüísticos como extralingüísticos. Se trata de un conjunto de rasgos discretos que pueden ser activados con mayor o menor intensidad en función de los contextos y en virtud de los procesos atencionales desencadenados en cada contexto.

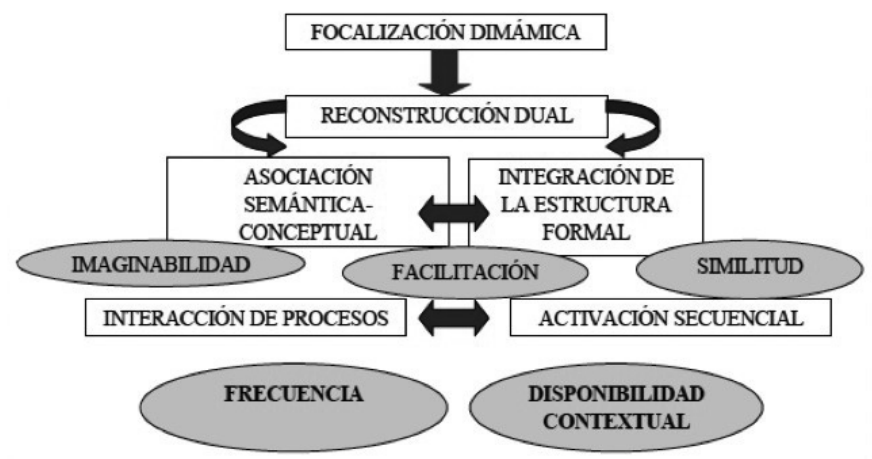

Modelo multifactorial del procesamiento léxico

Como se observa en la figura superior, nos inclinamos hacia un modelo dual que integra un sistema de análisis semántico-conceptual, que interactúa durante el procesamiento con otros sistemas de reconstrucción de la estructura formal de la palabra, todos ellos dirigidos por el mecanismo cognitivo de la focalización dinámica y afectados por múltiples efectos relacionados con la experiencia lingüística acumulada por el interpretante y el entorno contextual que envuelve la comunicación, entre los que se hallan la imaginabilidad conceptual, la facilitación y la activación secuencial, pero sobre todo la frecuencia y la disponibilidad contextual. Consideramos, además, que este marco explicativo resulta de sumo interés desde la óptica de la producción léxica, a la hora de describir fenómenos como los estados de la punta de la lengua (o TOT, del inglés tip of tongue) y los errores espontáneos del habla, en los que se produce un reclutamiento insuficiente o desviado de rasgos en el proceso de construcción léxica. 


\section{PRINCIPIOS ORGANIZADORES DEL CONOCIMIENTO LÉXICO. Similitud Y CONTRASTE}

A través de la aproximación psicolingüística al conocimiento léxico se ha tratado de dilucidar cómo se combinan e integran diferentes informaciones reunidas a través de distintas vías y experiencias para crear una representación significativa. Si consideramos, como hemos expuesto más arriba, que durante el procesamiento el individuo se apoya en un mecanismo de reconstrucción léxica, vinculado a sistemas de análisis secuencial y guiado por factores como la frecuencia y el marco contextual, merece la pena reflexionar sobre los mecanismos mentales responsables de la organización de tales conocimientos. De acuerdo con este propósito, nos interesa destacar aquí la actuación de los principios de similitud y contraste, no solo por su trascendencia desde el punto de vista de la agrupación de los rasgos de cada palabra y en los procesos de construcción de los significados, sino también por su relevancia tanto en los postulados teóricos clásicos de la semántica estructuralista como en los desarrollos más recientes de la Psicolingüística evolutiva y experimental (ver Varo Varo 2017).

La observación de estos principios y la relación entre ambos desde el punto de vista de la configuración del conocimiento lingüístico cuenta con una larga tradición en los estudios léxicos. En el marco de los trabajos fundacionales de la Semántica moderna como ciencia de la significación, queremos destacar, dentro de la orientación característica de la Lingüística de la época, las aportaciones de Bréal (1883,132-42 y 1897, 30), en relación con el funcionamiento de las por él denominadas leyes intelectuales del lenguaje. Estas ponen de relieve la inestabilidad de los rasgos de similitud plena y la tendencia a la búsqueda de rasgos de contraste por parte de los hablantes, por entrar en contradicción con la economía lingüística y cognitiva, inclinada al abandono del estado de identidad perfecta de las palabras. Asimismo, el principio de contraste constituye el fundamento del concepto de oposición elaborado por el estructuralismo lingüístico desde principios del siglo XX, al permitir definir cada unidad funcional del sistema en virtud de su análisis en rasgos distintivos. En concreto, es el lingüista ginebrino Ferdinand de Saussure, precursor de esta corriente, el primero en proponer una aproximación sistemática de las oposiciones que emanan de las lenguas, teniendo en cuenta que cada elemento, entendido como entidad discreta, puede ser definido negativamente o por sus diferencias respecto a los demás 
(160-61). La noción de negatividad adquiere tal magnitud en el Curso de Lingiuística General que llevará a una reformulación en el seno de la Escuela de Praga por parte de Karčevskij (88-93), que se extenderá al estructuralismo posterior, de modo que se reconoce la necesidad de que, junto a los rasgos diferenciales, exista una base común.

En cuanto a los modelos de la categorización, la adquisición y el procesamiento léxicos, ambos principios también encuentran eco, aunque son valorados de manera dispar y, en general, partiendo de una organización jerárquica de las categorías, se otorga en ellos un mayor protagonismo a los rasgos de similitud (ver Rosch y otros, Quinn; Keil; Mandler). A este respecto, los logros más recientes en la indagación de las bases cognitivas del lenguaje han demostrado que la clasificación en forma de jerarquía no es la única modalidad de estructuración de los conceptos (ver Murphy). El reconocimiento de la existencia de sistemas de clasificación $\operatorname{cruzados}^{19}$ justifica que una misma entidad pueda ser incluida en diferentes categorías semánticas, en función de los rasgos que se tomen como referencia. Junto a ello, se ha resaltado que la fuerza asociativa depende de cómo interactúen los significados en los contextos y de los rasgos experienciales de los referentes implicados, con lo que no solo permite diversas categorizaciones simultáneas y explica el aprendizaje (ver Di Giacomo y otros), sino que también contempla la flexibilidad en el protagonismo mayor o menor de los rasgos de similitud y contraste.

Desde nuestro punto de vista, la valoración de los principios de similitud y contraste a través de tareas de asociación semántica, al funcionar cada unidad léxica como un foco de atracción de relaciones sinonímicas y antonímicas sujetas a factores como la frecuencia y el contexto, permite abordar desde una perspectiva objetiva la relevancia de estos principios durante el procesamiento lingüístico. En este sentido, la eficiencia del principio de contraste, frente al de similitud, puede constatarse a través de simples tareas de asociación antonímica en la que los individuos participantes proporcionan tales respuestas para determinadas unidades léxicas incluidas en enunciados concretos (ver Varo Varo 2017). En estas pruebas es posible introducir desde marcos semánticamente apenas diferenciados hasta marcos antagónicos, para analizar la influencia del contexto lingüístico en el proceso de decisión antonímica. Asimismo, es posible considerar el tiempo de reacción para responder, con objeto

19. Desde la neuropsicología cognitiva se habla de sistemas de representación semántica múltiples (ver Mahon y Caramazza). 
de valorar la dificultad en el establecimiento de la relación léxica solicitada, así como la cohesión o dispersión semántica de los datos obtenidos, atendiendo a las primeras respuestas proporcionadas.

CONTEXTO 1 (C1) CONTEXTO 2 (C2)

Ella tomó la mano que le tendieron. Luis tomó sus libros y se marchó.

Has sido muy duro con ella.

Sus palabras fueron duras y me dolió.

Siento gran alegría por ser la elegida. Es una alegría que vengas a verme.

Ejemplo: prueba de asociación antonímica (tomado de Varo Varo 2017)

La diferencia en cuanto al procesamiento de las relaciones de similitud y contraste sugiere que la producción y la comprensión de las primeras parecen requerir mayor esfuerzo cognitivo que en el caso del contraste, por ser este último principio mucho más efectivo, al menos en la dinámica del hablar. Estos datos pueden correlacionarse con los proporcionados por la investigación de la ontogénesis de las relaciones léxicas, ${ }^{20}$ en los que se constata que la adquisición de la antonimia suele preceder a la de las otras relaciones como la hiperonimia-hiponimia y la parasinonimia (ver Varo Varo 2010). A la luz de estas observaciones, la antonimia puede interpretarse como relación entre dos dominios simétricos o un dominio escindido en partes simétricas, la hiperonimia-hiponimia como relación de inclusión de dominios y la parasinonimia (sustentada en la equipolencia semántica) como relación entre dominios solapados. En este sentido, dos parejas léxicas como "grande" / "pequeño" y "hombre" / "mujer" podrían analizarse como relación entre dos partes simétricas de los dominios 'dimensión' y 'ser humano'. En cambio, en los pares "mueble" / "silla" y "oír" / "escuchar" se analizaría el primer elemento como representante de un dominio incluido en el dominio representado en el segundo. Finalmente, los casos de pares como "joven" / "nuevo" y "hospital" / "clínica" ejemplifican dominios solapados o coincidentes en una parte de su esfera conceptual.

20. La ontogénesis de la antonimia es abordada en Varo Varo (2003, 16-27). 


\begin{tabular}{|l|l|}
\hline GRANDE & PEQUEÑO \\
\hline
\end{tabular}

OÍR

ESCUCHAR

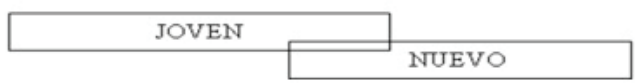

Representación de las diferentes relaciones semánticas: dominio escindido ("grande"-“pequeño"), inclusión de dominios (“oír”-“escuchar”) y dominios solapados (“joven”-“nuevo”)

Paralelamente, desde la perspectiva de las bases biológicas del lenguaje, mediante técnicas de neuroimagen se ha verificado la relación de determinadas capacidades para el establecimiento de oposiciones por contraste ${ }^{21}$ con ciertas áreas cerebrales, como el giro angular (ver Butters, Barton y Brody; Caramazza y otros 1976). Semejantes conclusiones pueden extraerse del estudio del procesamiento de tales relaciones en casos de afasia cerebral, que suelen atribuir distinto grado de complejidad a sinonimia y antonimia. Gardner y otros colegas (301-17) hace ya algunas décadas realizaron estudios de la comprensión y producción de antónimos en pacientes afásicos, con objeto de demostrar la existencia de una estructura interna previa al dominio significativo que se manifiesta a través de una estructura lingüística o simbólica. En ese sentido, el principio de contraste serviría de eje vertebrador no solo de diversas categorías lingüísticas, sino también de distintas redes de relaciones semánticas.

Asimismo, el análisis de conjunto de las relaciones de similitud y contraste presentes en el léxico de las lenguas se puede afrontar a partir de la

21. En la investigación de los procesos conceptuales y asociativos vinculados a las relaciones léxicas, parte de las conclusiones se basan en el análisis neuronal de las respuestas manifestadas ante determinados estímulos (como los realizados por Vaughan y otros y Herrman y otros), que pretenden descubrir qué elementos intervienen cognitivamente en su reconocimiento. Igualmente podemos mencionar a este respecto otros trabajos experimentales sobre el reconocimiento de sinónimos y antónimos (como el de Gazzaniga y Miller), junto con aproximaciones desde el punto de vista nocional o asociativo a su funcionamiento (ver Gross y otros; Berthonneau; Murphy y Andrew; Charles y otros; Sabourin y Libben), que coinciden en señalar notables diferencias. 
actuación de procesos cognitivos atencionales, más arriba comentados, que nos permiten señalar ciertas diferencias patentes tanto en el conocimiento sobre el léxico como en su uso (ver Varo Varo 2010). Así, similitud y contraste constituyen principios de base cognitiva, que muestran una desigual actuación, en la que parece tener preeminencia el segundo, se activan a través de la habilidad cognitiva de focalización y se proyectan sobre el lenguaje, por lo que se convierten en un mecanismo fundamental para la configuración del conocimiento léxico y el acceso a este. Sin embargo, como puede extraerse de los datos experimentales, tal configuración no es estática, sino que se ve afectada por otros aspectos como la frecuencia y el contexto.

\begin{tabular}{lllll}
$\begin{array}{l}\text { RELACIÓN } \\
\text { LÉXICA }\end{array}$ & $\begin{array}{l}\text { CONFIGURACIÓN } \\
\text { DOMINIOS }\end{array}$ & ADQUISICIÓN & PROCESAMIENTO & FOCALIZACIÓN \\
\hline Antonimia & $\begin{array}{l}\text { Dominio } \\
\text { escindido }\end{array}$ & $\begin{array}{l}\text { Más } \\
\text { temprana }\end{array}$ & $\begin{array}{l}\text { Menor tiempo } \\
\text { de procesamiento }\end{array}$ & Contraste \\
\hline $\begin{array}{l}\text { Hiperonimia- } \\
\text { hiponimia }\end{array}$ & $\begin{array}{l}\text { Inclusión } \\
\text { de dominios }\end{array}$ & Más tardía & $\begin{array}{l}\text { Mayor tiempo } \\
\text { de procesamiento }\end{array}$ & Similitud \\
\hline Parasinonimia & $\begin{array}{l}\text { Dominios } \\
\text { solapados }\end{array}$ & Más tardía & $\begin{array}{l}\text { Mayor tiempo } \\
\text { de procesamiento }\end{array}$ & $\begin{array}{l}\text { Similitud / } \\
\text { Contraste }\end{array}$ \\
\hline
\end{tabular}

Aspectos diferenciales entre las relaciones léxicas desde la perspectiva psicolingüística

\section{CONCLUSIONES}

Como conclusión, la Semántica léxica no puede desarrollarse a espaldas de los avances en neurociencia, que nos proporcionan evidencias empíricas abundantes sobre dónde, cuándo y cómo se procesa el significado de las palabras. Ahora bien, también estos últimos pueden beneficiarse, sin duda, de los logros alcanzados en la investigación lingüística dirigida a otorgar al contenido léxico un estatus bien definido.

Como resultado de las observaciones anteriores, fundamentalmente derivadas del análisis de los déficits semánticos, la neología semántica y el procesamiento de las relaciones léxico-semánticas, es posible inferir la necesidad de interpretar el significado léxico como una agrupación de rasgos semánticos discretos basada en la frecuencia asociativa y, por tanto, en la experiencia lingüística del individuo, que debe ser diferenciada, sin renunciar a sus conexiones, de la información conceptual vinculada a la palabra y que se ve sujeta a los 
principios organizadores de similitud y contraste. Este enfoque no solo puede servir de punto de referencia para discutir los actuales modelos elaborados para explicar fenómenos léxicos como los que hemos comentado aquí, sino también el papel del contenido lingüístico en la comprensión y la producción léxicas. Para ello, se debe seguir profundizando en la situación del significado en conexión con el conocimiento léxico y completar este análisis con la indagación de correlatos neurológicos adecuados a los resultados obtenidos de la investigación psicolingüística.

\section{OBRAS CITADAS}

Agosta, Federica, y otros. "Language networks in semantic dementia". Brain. Fournal of Neurology 133 (2010): 286-99.

Ahrens, Kathleen, y otros. "Functional MRI of conventional and anomalous metaphors in Mandarin Chinese". Brain and Language 100 (2007): 163-71.

Allport, D. Alan. "Language and cognition". Approaches to language. Ed. Roy Harris. Oxford: Pergamon Press, 1983. 61-94.

Arzouan, Yossi, y otros. "Dynamics of hemisphere activity during metaphor comprehension: electrophisiological measures". NeuroImage 36 (2007): 222-51.

Barsalou, Lawrence W. "Perceptual and symbol systems". Behavioral and Brain Sciences 22 (1999): 577-660.

Barsalou, Lawrence W. “Grounded Cognition”. Annual Review of Psychology 59 (2008): 617-45.

Bennett, Max R., y Peter M. S. Hacker. "Language and cortical function: conceptual developments". Progress in Neurobiology 80 (2006): 20-52.

Berthonneau, Anne-Marie. "Avant/après: de l'espace au temps". Lexique 11 (1993): 41-109.

Binder, Jeffrey R., y otros. "Human temporal lobe activation by speech and nonspeech sounds". Cerebral Cortex 10 (2000): 512-28.

Bottini, Gabriella, y otros. "The role of the right hemisphere in the interpretation of figurative aspects of language: a positron emission tomography activation study". Brain 117 (1994): 1241-53.

Bozeat, Sasha, y otros. "Non-verbal semantic impairment in semantic dementia". Neuropsychologi, 38 (2000): 1207-15.

Bradley, Dianne C., y Kenneth I. Forster. "A reader's view of listening”. Cognition 25 (1987): 103-34. 
Bréal, Michel. "Les lois intellectuelles du langage. Fragment de sémantique". Annuaire de l'Association pour l'encouragement des études grecques en France. Paris: Maisonneuve et Cie/ Libraires-Éditeurs, 1987. 132-42.

Bréal, Michel. Essai de sémantique. Science des significations. 7. a ed. 1924. Paris: Librairie Hachette, 1897.

Burani, Cristina, y Alfonso Caramazza. "Representation and processing of derived words", Language and Cognitive Processes 2 (1987): 217-27.

Butters, Nelson, Melvin Barton y Betty A. Brody. "Role of the right parietal lobe in the mediation of cross modal associations and reversible operations in space". Cortex 6 (1970): 174-90.

Caramazza, Alfonso, y otros. "Right hemisphere damage and verbal problem solving"; Brain and language 3 (1976): 41-46.

Caramazza, Alfonso, y otros. "The multiple semantics hypothesis: multiple confusion?". Cognitive Neuropsychology 7 (1990): 161-89.

Caramazza, Alfonso, y Bradford Z. Mahon. "The organization of conceptual knowledge: the evidence from category-specific semantic deficits". Trends in Cognitive Sciences 7.8 (2003): 354-61.

Casas Gómez, Miguel. "Implicaciones léxicas de los niveles del significar". Panorama der Lexikalischen Semantik Thematische Festschrift aus Anlab des 60. Geburtstags von Horst Geckeler. Ed. Ulrich Hoinkes. Tübingen: Gunter Narr, 1995. 101-12.

Casas Gómez, Miguel. Las relaciones léxicas. Tübingen: Niemeyer, 1999.

Casas Gómez, Miguel. Los niveles del significar. Documentos de Investigación Lingüística 7. Cádiz: Universidad de Cádiz, 2002.

Casas Gómez, Miguel. "A typology of Relationships in Semantics”. Quaderni di Semántica 35.2 (2014): 45-73.

Charles, Walter G., y otros. "Conceptual and associative processing in antonymy and synonymy”. Applied Psycholinguistics 15.3 (1994): 329-54.

Cree, George S., Ken McRae y Chris McNorgan. "An attractor model of lexical conceptual processing: simulating semantic priming". Cognitive Science 23.3 (1999): 371-414.

Croft, William, y D. Alan Cruse. Cognitive Linguistics. Cambridge, UK: Cambrigde University Press, 2004.

Cuetos, Fernando. Anomia: la dificultad para recordar las palabras. Madrid: TEA, 2003.

Di Giacomo, Dina, y otros. "Semantic associative relations and conceptual processing”. Cognitive Processing 13 (2012): 55-62. 
Díaz Hormigo, $M^{\mathrm{a}}$ Tadea, y Carmen Varo Varo. "Neology and Cognition". Linguistics Insights. Vol. 158. Studies in Linguistics and Cognition. Ed. Bárbara Eizaga Rebollar. Bern: Peter Lang, 2012. 15-34.

Diéguez Vide, Faustino, y Jordi Peña-Casanova. Cerebro y lenguaje. Sintomatología neurolinguiística. Madrid: Médica Panamericana, 2012.

Donoso, Archibaldo, y otros. "Demencia semántica. Presentación de dos casos y revisión de la literatura". Revista Chilena de Neuro-Psiquiatría 46.4 (2008): 280-87.

Ellis, Andrew W. "The production of spoken words: A cognitive neuropsychological perspective". Progress in the Psychology of Language. Ed. Andrew W. Ellis. Vol. 2. London: Lawrence Erlbaum Associates, 1985. 107-45. Versión en español "La producción de palabras habladas desde la perspectiva de la neuropsicología cognitiva”. Lecturas de Psicolingiǘstica. 2. Neuropsicología cognitiva del lenguaje. Eds. Francisco Valle, Fernando Cuetos, José Manuel Igoa y Susana del Viso. Madrid: Alianza, 1990. 125-57.

Farah, Martha, y Murray Grossman. "Semantic memory impairments". Behavioral neurology and neuropsychology. Eds. Todd E. Feinberg y Martha J. Farah. $2^{a}$ ed. McGraw Hill: New York, 1997. 473-77.

Forster, Kennteth, y Susan M. Chambers. "Lexical access and naming time". Fournal of Verbal Learning and Verbal Bebaviour 12 (1973): 62735 .

Gardner, Howard, y otros. "The Appreciation of Antonymic Contrasts in Aphasia". Brain and Language 6.3 (1978): 301-17.

Gazzaniga, Michael S., y George A. Miller. "The Recognition of Antonymy by a Language-Enriched Right Hemisphere”. Fournal of Cognitive Neuroscience 1.2 (1989): 187-93.

Geiger, Odie. "Metaphors and the Mental Lexicon". Brain and Language 68 (1999): 190-98.

Glenberg, Arthur M., y Vittorio Gallese. "Action-based Language: a theory of language acquisition, comprehension, and production". Cortex 48 (2012): 905-22.

Glucksberg, Sam. "The psycholinguistics of metaphor". Trends in Cognitive Science 7 (2003): 92-96.

Goodglass, Harold, y Arthur Wingfield, eds. Anomia: neuroanatomical and cognitive correlates. New York: Academic Press, 1997.

Gorno-Tempini, Maria Luisa, y otros. "Clinical, cognitive and anatomical 
evolution from nonfluent progressive aphasia to corticobasal syndrome; a case report". Neurocase 10 (2004): 426-36.

Gross, Derek, y otros. "The Organization of Adjectival Meanings". Fournal of Memory and Language 28.1 (1989): 92-106.

Gutiérrez Ordóñez, Salvador. Lingüística y Semántica: aproximación funcional. Oviedo: Universidad de Oviedo, 1981.

Gutiérrez Ordóñez, Salvador. Introducción a la semántica funcional. Madrid: Síntesis, 1989.

Hebb, Donald. The Organization of Behavior: a Neuropsychological Theory. New York: John Wiley \& Sons, 1949.

Herrmann, Douglas J., y otros. "The Role of Elements of Relation Definition in Antonym and Synonym Comprehension". Zeitschrift für Psychologie mit Zeitschrift für angewandte Psychologie 194.2 (1986): 133-53.

Hickok, Gregory, y David Poeppel. "Dorsal and ventral streams: a framework for understanding aspects of the functional anatomy of language". Cognition 92 (2004): 67-99.

Hickok, Gregory, y David Poeppel. "The cortical organization of speech processing”. Nature Reviews Neuroscience 8 (2007): 393-402.

Hodges, John R., y Karalyn Patterson. "Semantic dementia: a unique clinicopathological syndrome”. Lancet Neurology 6.11 (2007): 1004-14.

Karčevskij, Sergej. "Du dualisme asymétrique du signe linguistique". Travaux du Cercle Linguistique de Prague 1 (1929): 88-93.

Keil, Frank C. "The primal frontier? Spatial cognition and the origin of concepts”. Philosophical Psychology 21.2 (2008): 241-50.

Kiefer, Markus, y Friedemann Pulvermüller. "Conceptual representations in mind and brain: theoretical developments, current evidence and future directions". Cortex 48 (2012): 805-25.

Kutas, Marta, y Kara Federmeier. "Electrophysiology reveals semantic memory use in language comprehension". Trends in Cognitive Sciences 4.12 (2000): 463-70.

Lai, Vicky, Tim Curra, y Lise Menn. "Comprehending conventional and novel metaphors: an ERP study". Brain Research 1284 (2009): 145-55.

Lakoff, George. "The contemporary theory of metaphor". Metaphor and Thought. Ed. Andrew Ortony. Cambridge: Cambridge University Press, 1993. 202-51.

Lakoff, George, y Mark Johnson. Metaphors we live by. Chicago, IL: University of Chicago Press, 1980. 
Lamb, Sydney. Pathways of the brain. The neurocognitive basis of language. Amsterdam/Philadelphia: John Benjamins, 1999.

Lamb, Sydney. Jonathan Webster, eds. Language and reality: selected writings of Sydney Lamb. London: Continuum International Publishing, 2004.

Lambon Ralph, Mathew A. y otros. "Naming in dementia-what matters?". Neuropsychologia 36.8 (1998): 775-84.

Mahon, Bradford, y Alfonso Caramazza. "Concepts and categories: a cognitive neuropsychological perspective". Annual Review of Psychology 60 (2009): 27-51.

Mandler, Jean M. "On the birth and growth of concepts". Philosophical Psychology 21.2 (2008): 207-30.

Marangolo, Paola, y otros. "The right hemisphere involvement in morphologically derived words". Fournal of Cognitive Neuroscience 15 (2003): 364-71.

Marchetti, Giorgio. "A presentation of Attentional Semantics”. Cognitive Processing, 7.13 (2006), 163-94.

Marchetti, Giorgio, Giulio Benedetti y Ahlam Alharbi, eds. Attention and Meaning. The Attentional Basis of Meaning. New York: Nova Publishers, 2015.

Mashal, Nira, y otros. "An fMRI investigation of the neural correlates underlying processing of novel metaphoric expressions". Brain and Language 100 (2007): 115-26.

McGlone, Mathew, y Deanna Manfredi. "Topic-vehicle interaction in metaphor comprehension". Memory and Cognition 29 (2001): 1209-19.

Morton, John. "Interaction of information in word recognition". Psychological Review 76 (1969): 165-78.

Morton, John. "Facilitation in word recognition: Experiments causing change in the logogen model". Processing of visible language. Eds. Paul A. Kolers, Merald E. Wrolstad y Herman Bouma. Vol. 1. New York: Plenum, 1979. 259-68.

Murphy, Gregory L. The big book of concepts. Cambridge, Ma.: MIT Press, 2004. Murphy, Gregory L., y Jane M. Andrew. "The conceptual Basis of Antonymy and Synonymy in Adjectives". Fournal of Memory and Language 32.3 (1993): 301-19.

Patterson, Karalyn, Peter J. Nestor y Timothy Rogers. "Where do you know what you know? The representation of semantic knowledge in the human brain". Nature 8 (2007): 976-87.

Pollio, Howard K., y otros. "Need metaphoric comprehension take longer 
than literal comprehension?". Fournal of Psycholinguistic Research 13.3 (1984): 195-214.

Pulvermüller, Friedemann. "Brain reflections of words and their meanings". Trends in Cognitive Sciences 5.12 (2001): 217-24.

Pulvermüller, Friedemann. The neuroscience of language. Reimpr. Cambridge: Cambridge University Press, 2005.

Pulvermüller, Friedemann. "Meaning and the brain: the neurosemantics of referential, interactive, and combinatorial knowledge". Fournal of Neurolinguistics 25 (2012): 423-59.

Pulvermüller, Friedemann. "How neurons make meaning: brain mechanisms for embodied and abstract-symbolic semantics". Trends in Cognitive Sciences 17.9 (2013): 458-70.

Pynte, Joe, y otros. "The time-course of metaphor comprehension: an eventrelated potential study". Brain and Language 55 (1996): 293-316.

Quinn, Paul C. "Development of Subordinate-Level Categorization in 3-to 7 Month-Old Infants”. Child Development 75 (2004): 886-99.

Rivano, Emilio. Metáfora y linguistica cognitiva. Santiago de Chile: Bravo y Allende Editores, 1997.

Rosch, Eleanor, y otros. "Basic objects in natural categories". Cognitive Psycho$\log y 8$ (1976): 382-439.

Rumelhart, David, y James McClelland. Parallel Distributed Processing: explorations in the microstructure of cognition. Cambridge: MIT Press, 1986.

Sabourin, Laura, y Gary Libben. "Lexical Processing of Synonymy and Antonymy: an Exploration of Task and Word Form Differences". University of Alberta Papers in Experimental and Theoretical Linguistics 5 (2000): 114-36.

Saussure, Ferdinand de. Cours de linguistique générale. Publié par Ch. Bally et A. Sechehaye avec la collaboration de A. Riedlinger. $2^{a}$ ed. Paris: Payot, 1922.

Schmidt, Gwen, y otros. "Right hemisphere metaphor processing? Characterizing the lateralization of semantic processes". Brain and Language 100 (2005): 127-41.

Shibata, Midori, y otros. "Neural Mechanims involved in the comprehension of metaphoric and literal sentences: an fMRI study". Brain Research 1166 (2007): 92-102.

Snowden, Julie S., Peter J. Goulding y David Neary. "Semantic dementia: a form of circumscribed cerebral atrophy". Bebavioral Neurology 2 (1989): 167-82.

Thibodeau, Paul, y Frank H. Durgin. "Productive figurative communication: 
conventional metaphors facilitate the comprehension of related novel metaphors". Fournal of Memory and Language 58 (2008): 521-40.

Ullmann, Michael T. "Contributions of memory circuits to language: the declarative/procedural model”. Cognition 92.1-2 (2004): 231-70.

Varo Varo, Carmen. La polaridad en el lenguaje. Documentos de Investigación Lingüística 8. Cádiz: Universidad de Cádiz, 2003.

Varo Varo, Carmen. "El procesamiento de las relaciones léxicas". Revista Española de Lingiuística 40.1 (2010): 149-71.

Varo Varo, Carmen. "Aproximación teórico-práctica al procesamiento lingüístico de neologismos léxicos”. Revista Signos. Estudios de Lingüística 46.81 (2013): 132-52.

Varo Varo, Carmen. "La configuración mental del significado: evidencias a partir de la anomia". Avances en Lingüistica Clínica. Eds. Ignacio MorenoTorres Sánchez, Esther Moruno López y Sonia Madrid Cánovas. Málaga: Publicaciones y Divulgación Científica de la Universidad de Málaga, 2014. 73-80.

Varo Varo, Carmen. "El estudio de los déficits semánticos al servicio de la teoría del significado". Quaderni di Semantica. Nuova serie 1 (2015): 77 97.

Varo Varo, Carmen. "Los principios de similitud y contraste en el procesamiento léxico: aproximación teórico-práctica". Anuario de Letras 5.2 (2017). En prensa.

Varo Varo, Carmen, $M^{a}$ Tadea Díaz Hormigo y $M^{a}$ Jesús Paredes Duarte. "Modelos comunicativos y producción e interpretación neológicas". Revista de Investigación Linguiistica 12 (2009): 185-216.

Vaughan, Jonathan, y otros. "Cortical Evoked Responses to Synonyms and Antonyms". Memory and Cognition 10.3 (1982): 225-31. 

La teoría semántica en

los estudios de casos 
